\title{
Cancer chemoprevention approaches
}

\author{
Capek I* \\ Slovak Academy of Sciences, Institute of Measurement Sciences, Bratislava, Slovakia
}

\begin{abstract}
Chemopreventive agents exhibit antioxidative and antiproliferative activities, signal transduction pathways, cell cycle, etc., and affect the process of carcinogenesis and modulate enzyme activity. They inhibit carcinogenesis at initiation phase or at promotion/progression stage. Chemoprotective components can be nutrients like, resistant starch, non-starch polysaccharides, oligosaccharides, folates, selenium, curcumin, zinc, or nonnutritive bioactive constituents such as protease inhibitors, saponins, phytosterols, lectins and phytates. These natural products have a low toxicity, affect NFKB activity, stimulate immune cell activity and inhibit IKK activity.
\end{abstract}

Cancer is a disease that is associated with genetic lesions and epigenetic modifications. During oncogenesis and malignant cellular transformation, DNA undergoes significant changes in methylation, resulting in both activation and silencing of genes. Particularly genes involved in cell cycle regulation, cell growth, and differentiation are affected leading to uncontrolled expansion of cancerous cells. Cancers are classified mainly in three groups, carcinomas, leukemias/ lymphomas, and sarcomas, based on their tissue origin during embryonic development. About $90 \%$ of the cancers in humans are malignancies of epithelial cells, the carcinomas. Leukemias and lymphomas arise from the hematopoietic cells of bone marrow and are responsible for roughly $8 \%$ of human cancers. Whereas leukemias tend to proliferate as single cells, lymphomas grow as aggregates of tumor cells. Sarcomas, which are relatively rare in humans (approximately $1 \%$ of total cancer cases), are solid tumors of connective tissues $[1,2]$.

The cell cycle comprises four phases, gap 1 (G1), synthesis (S), mitosis (M) and gap $2(\mathrm{G} 2)$. Within the cycle there are three major checkpoints, G1 (verification of growth and environmental suitability for DNA synthesis, G2 (check on DNA synthesis and conditions for cell division) and finally metaphase (checking chromosome alignment on the spindle) [1].

An interesting approach for cancer treatment is referred as cancer chemoprevention which involves inhibition of carcinogenesis at initiation phase or at promotion/progression stage [3]. Chemopreventive agents are thus classified into two categories: blocking and suppressive agents. Blocking agents inhibit the activation of carcinogen through metabolic pathways and restrict the interaction of carcinogenic agents with biomolecules. Suppressive agents suppress promotion or progression of cancer cells [4]. Chemopreventive agents can have antioxidative and antiproliferative effects or they modulate enzyme activity, signal transduction pathways, cell cycle, etc., thus affecting the process of carcinogenesis [5].

A wide range of chemoprotective components are present in legumes. These are nutrients like, resistant starch, non-starch polysaccharides, oligosaccharides, folates, selenium, zinc, or nonnutritive bioactive constituents such as protease inhibitors, saponins, phytosterols, lectins and phytates [6]. Legume seeds are though the part of our diet but other parts of plants like roots, leaves, and barks are also traditionally used for various medicinal purposes. Many natural products affect NFkB activity, either directly or indirectly, and due to their low toxicity, they are good candidates for chemoprevention.

The main compounds responsible for this effect are polysaccharides and their connections to peptides (proteoglycans) or steroids $[7,8]$. Mushroom polysaccharides are one of the most known groups of mushroom compounds with anticancer activities, which can be both soluble and insoluble in water. Polysaccharides-decorated polymer and metal nanoparticles increase the solubility, bioavailability and biological activity of chemoprotective components. Polysaccharides are thpoght to activate the immune response in vitro and in vivo, acting as biological stimulants. The most important anticancer polysaccharides are made up of compounds with a structure similar to those which are components of the cell wall of mushrooms.

The mechanism of the immune system activity was confirmed in subsequent studies which involve stimulation of immune system cells, including T lymphocytes and cytotoxic Tlymphocytes (CTL), B lymphocytes, granulocytes (eosinophils and neutrophils), natural killers (NK), or macrophages. This mechanism is particularly characteristic for $\beta$-1,3-glucans, but numerous studies also suggest that $\beta$-glucans may enhance the specific cellular response by enhancing the secretion of IL-6, IL-8, IL-12, and IFN-Y from neutrophils, macrophages, and NK cells $[7,8]$. Moreover, $\beta$-glucans found in the fruiting bodies of edible mushrooms may constitute the factors that stimulate new effector cells contributing to the formation of, inter alia, antibodies directed against cancer antigens, which is less popular than the classic cytotoxic effect caused by chemotherapy [8].

The therapeutic effect is primarily due to the synergistic polysaccharide-lentinan interaction, whose action causes a reduction in tumor size even by $90 \%$ [7]. This polysaccharide is most commonly used to treat solid tumors of stomach, lung, breast, colon, and malignant

${ }^{\star}$ Correspondence to: Ignác Capek, Slovak Academy of Sciences, Institute of Measurement Sciences, Bratislava, Slovakia, E-mail: Ignac.Capek@savba.sk

Key words: chemoprevention, immunity, cancer, nutrients, polyphenols, decoration, polysaccharide

Received: November 02, 2019; Accepted: November 20, 2019; Published: November 25, 2019 
leukemia. Lentinan does not exhibit cytotoxic activity, practically does not demonstrate any side effects, only the ones such as local irritation after injection or sporadically observed fever and vomiting, but usually these are only the episodes, and what the most important, it is generally well tolerated by the patient's organism. It extends an average time of the survival of patients with cancer in whom it is used.

$\beta$-glucan from $G$. frondosa exhibited cytotoxic effect on human prostate cancer cells (PC-3 cells). Induction of apoptosis was confirmed in vitro for adrogen-independent tumor [8]. It was also demonstrated in the studies that polysaccharide isolated from another species-Pleurotus pulmonarius-is effective in liver cancer treatment, both in vitro and in vivo. This activity involved an inhibition of cancer development via inhibiting effect on VEGF-induced (vascular endothelial growth factor) PI3K/AKT signaling pathway [9].

The mushroom polysaccharides were observed to prevent cancers, and in case of already detected cancers, exhibit its cell cycle inhibition and apoptosis induction, affect cancer cell growth and development (growth reduction even up to 50\%), as well as an inhibition of metastases formation, prolonging patient's survival [10].

Green chemoprevention is a term used to coin interventions that utilize derivatives of natural compounds such as curcumins, quercetin resveratrol, green tea extract, luteolin, soybeans, and pomegranate. All these aforementioned plant products have antioxidant properties secondary to the inherent polyphenols present in them [11]. Polyphenol is a subgroup of phytochemicals which inhibit cancer development through different strategies, constrain the intracellular oncoprotein signaling along with the PKC/RAS/ MPK or p13-kinase/AKT pathways, downregulate the pro-proliferative anti-apoptotic transcription factors $\mathrm{NF \kappa B}$ and $\mathrm{AP}-1$, and upregulate the carcinogen-detoxifying enzymes and DNA repair proteins [12].

Curcumin is a polyphenol derived from turmeric (Curuma longa). In the laboratory, curcumin inhibits NFkB activity in ovarian, breast, head and neck, lung, and prostate cancer cell lines [13]. In the clinic, curcumin is well tolerated and has some biological activity in phase II pancreatic cancer trials [14]. Resveratrol is a polyphenol derived from red grapes and berries. Like curcumin, a significant amount of in vitro data has shown that resveratrol inhibits the growth of multiple cancer cell lines including the breast, prostate, thyroid, head and neck, ovarian, and cervical. Resveratrol appears to regulate cancer cell growth by inhibition of IKK and suppression of NFKB activity [15]. Resveratrol also inhibits IKK activity in animal models of colitis. Data from in vivo preclinical trials shows that resveratrol can prevent tumor growth or carcinogenesis in several cancer sites including the breast, skin, prostate, gastrointestinal, and lung. Clinical trials to date, which are mostly risk assessment studies, show that resveratrol-rich products may be beneficial for cancer prevention.

\section{Conclusion}

Chemopreventive agents are reported to have antioxidative and antiproliferative effects, modulate enzyme activity, signal transduction pathways, cell cycle, etc., and affect the process of carcinogenesis. They inhibit carcinogenesis at initiation phase or at promotion/progression stage. Green chemoprevention is a term used to coin interventions that utilize derivatives of natural compounds such as curcumins, resveratrol, green tea extract, luteolin, soybeans, and pomegranate. They inhibit cancer cell cycle development and stimulate the cancer cell apoptosis.

\section{References}

1. Cooper G (2000) The cell: A molecular approach. Sinauer Associates Inc. Sunderland, MA.

2. Goldsby RA, Kindt TJ, Kube J, Osborne BA (2002) Immunology. W. H. Freeman \& Company, New York, NY.

3. Sporn MB, Dunlop NM, Newton DL, Smith JM (1976) Prevention of chemical carcinogenesis by vitamin a and its synthetic analogs. Fed Proc 35: 1332-1338. [Crossref]

4. Wattenberg LW (1985) Chemoprevention of cancer. Cancer Res 45: 1-8. [Crossref]

5. Steward WP, Brown K (2013) Cancer chemoprevention: a rapidly evolving field. $\mathrm{Br} J$ Cancer 109: 1-7. [Crossref]

6. Mathers JC (2002) Pulses and carcinogenesis: potential for the prevention of colon breast and other cancers. Br J Nutr 88: 273-279. [Crossref]

7. Meng X, Liang H, Luo L (2016) Antitumor polysaccharides from mushrooms: a review on the structural characteristics, antitumor mechanisms and immunomodulating activities. Carbohydr Res 424: 30-41. [Crossref]

8. Singdevsachan SK, Auroshree P, Mishra J, Baliyarsingh B, Tayung K, et al. (2016) Mushroom polysaccharides as potential prebiotics with their antitumor and immunomodulating properties: a review. Bioact Carbohydr Dietary Fibre 7: 1-14

9. Cheung PCK (2013) Mini-review on edible mushrooms as source of dietary fiber: preparation and health benefits. Food Sci Human Wellness 2: 162-166.

10. Zong A, Cao H, Wang F (2012) Anticancer polysaccharides from natural resources: a review of recent research. Carbohydr Polym 90: 1395-1410. [Crossref]

11. Saba NF, Haigentz M Jr, Vermorken JB, Strojan P, Bossi P, et al. (2015) Prevention of head and neck squamous cell carcinoma: removing the "chemo" from "chemoprevention". Oral Oncol 51: 112-118. [Crossref]

12. Sheth SH, Johnson DE, Kensler TW, Bauman JE (2015) Chemoprevention targets for tobacco-related head and neck cancer: past lessons and future directions. Oral Oncol 51: 557-564.

13. Brown M, Cohen J, Arun P, Chen Z, Van Waes C (2008) NF-kappaB in carcinoma therapy and prevention. Expert Opin Ther Targets 12: 1109-1122. [Crossref]

14. Dhillon N, Aggarwal BB, Newman RA, Wolff RA, Kunnumakkara AB, et al. (2008) Phase II trial of curcumin in patients with advanced pancreatic cancer. Clin Cancer Res 14: 4491-4499. [Crossref]

15. Brown M, Cohen J, Arun P, Chen Z, Van Waes C (2008) NF-kappaB in carcinoma therapy and prevention. Expert Opin Ther Targets 12: 1109-1122. [Crossref]

Copyright: (C2019 Capek I. This is an open-access article distributed under the terms of the Creative Commons Attribution License, which permits unrestricted use, distribution, and reproduction in any medium, provided the original author and source are credited. 